\title{
Elevated preoperative plasma D-dimer levels and the incidence of venous thromboembolism in Japanese females with gynecological cancer
}

\author{
JUNICHI KODAMA ${ }^{1}$, NORIKO SEKI ${ }^{2}$, CHIKAKO FUKUSHIMA ${ }^{2}$, TOMOYUKI KUSUMOTO ${ }^{2}$, \\ KEIICHIRO NAKAMURA ${ }^{2}$, ATSUSHI HONGO ${ }^{2}$ and YUJI HIRAMATSU ${ }^{2}$ \\ ${ }^{1}$ Department of Obstetrics and Gynecology, Hiroshima City Hospital, Hiroshima 730-8518; \\ ${ }^{2}$ Department of Obstetrics and Gynecology, Okayama University Graduate School of Medicine, \\ Dentistry and Pharmaceutical Sciences, Okayama 700-8558, Japan
}

Received June 14, 2012; Accepted October 11, 2012

DOI: $10.3892 / \mathrm{ol} .2012 .970$

\begin{abstract}
The purpose of the present study was to determine the incidence of increased levels of D-dimer and associated factors in preoperative patients with gynecological cancer. Furthermore, we determined the incidence and risk factors associated with preoperative venous thromboembolism (VTE). Overall, 456 patients with invasive gynecological cancer scheduled to undergo surgery were recruited. Preoperative plasma D-dimer levels were measured and patients whose plasma D-dimer concentration exceeded the pre-set cut-off value underwent computed tomography scanning. The incidence of elevated D-dimer and VTE was identified as significantly higher in patients with ovarian cancer. Multivariate analysis revealed that advanced age, low hemoglobin levels and elevated C-reactive protein (CRP) levels were independent factors for preoperative elevations in plasma D-dimer levels. Advanced age was an independent risk factor for preoperative VTE. Massive ascites and the presence of co-morbidities were independent risk factors for preoperative VTE in ovarian cancer. Advanced age and stage were independent risk factors for preoperative VTE in endometrial cancer. Advanced age was an independent risk factor for preoperative VTE in cervical cancer. Plasma D-dimer levels and the incidence of preoperative VTE were higher in patients with ovarian cancer compared with those with other gynecological cancers. Advanced age, low hemoglobin levels and elevated CRP levels were significant factors associated with elevated plasma D-dimer levels and age was an independent risk factor for preoperative VTE in gynecological cancer.
\end{abstract}

Correspondence to: Dr Junichi Kodama, Department of Obstetrics and Gynecology, Hiroshima City Hospital, 7-33 Moto-machi, Naka-ku, Hiroshima 730-8518, Japan

E-mail: kodama@cc.okayama-u.ac.jp

Key words: gynecological cancer, venous thromboembolism, preoperative D-dimer

\section{Introduction}

Cancer is a widely accepted risk factor for venous thromboembolism (VTE) and, to a lesser extent, arterial thrombosis. This risk is attributed to numerous factors, including expression of prothrombotic factors by tumors, tumor compression of vessels, inflammatory host response to malignancies, immobility, surgery, indwelling central venous catheters and specific antitumor therapies (1). VTE is a common complication of surgery for gynecological cancer and individuals with gynecological malignancies are classified as the highest risk group (2,3). It has been reported that $90 \%$ of pulmonary thromboemboli (PTEs) result from deep venous thromboses (DVTs) (4). VTE may occur prior to surgery, due to the hypercoagulable state well-described in cancer patients $(3,4)$. Subclinical VTE may progress and only manifest clinically following surgery. Therefore, it is important to perform preoperative VTE screening for gynecological cancer patients in order to prevent postoperative symptomatic PTE.

D-dimer, a marker of a hypercoagulable state, is a stable end-product of fibrin degradation and its levels increase as a result of fibrin formation and fibrinolysis. Measurement of plasma D-dimer levels is often performed during screening for VTE.

The aim of the present study was to determine the incidence of increased D-dimer levels and the associated factors in preoperative patients with invasive gynecological cancer. Furthermore, we determined the incidence and risk factors for preoperative VTE.

\section{Materials and methods}

Study population. Overall, 456 patients with invasive gynecological cancer scheduled to undergo surgery at Okayama University Hospital were recruited between August 2005 and August 2011. The median age of the patients was 55 years (range, 23-85 years). A range of gynecological cancers were diagnosed: 114 ovarian, 198 endometrial and 144 cervical cancers. The study was approved by the ethics committee of Okayama University Hospital, Okayama, Japan. 
Table I. Analysis of factors associated with preoperative elevation of plasma D-dimer levels in patients with gynecological cancer.

\begin{tabular}{lccc}
\hline Variables & $\begin{array}{c}\text { D-dimer }<1.3 \mu \mathrm{g} / \mathrm{ml} \\
(\mathrm{n}=329)\end{array}$ & $\begin{array}{c}\text { D-dimer } \geq 1.3 \mu \mathrm{g} / \mathrm{ml} \\
(\mathrm{n}=127)\end{array}$ & P-value \\
\hline Age (years), median $($ range $)$ & $54(23-81)$ & $59(30-85)$ & $<0.0001$ \\
Body mass index $\left(\mathrm{kg} / \mathrm{m}^{2}\right)$, median (range) & $22.2(15.0-46.5)$ & $22.5(13.5-36.4)$ & 0.310 \\
Comorbidity, $\mathrm{n}(\%)$ & $89(27.1)$ & $40(31.5)$ & 0.345 \\
Smoking, $\mathrm{n}(\%)$ & $30(9.1)$ & $7(5.5)$ & 0.206 \\
Blood group, $\mathrm{n}(\%)$ & & & 0.019 \\
$\mathrm{O}$ & $92(28.0)$ & $22(17.3)$ & \\
Non-O & $237(72.0)$ & $105(82.7)$ & 0.0007 \\
Leukocyte $\left(/ \mathrm{mm}^{3}\right)$, median (range) & $6200(2090-18300)$ & $12.5(7.7-15.9)$ & $<0.0001$ \\
Hemoglobin $(\mathrm{g} / \mathrm{dl})$, median (range) & $13.0(2.9-15.7)$ & $30.3(19.8-89.1)$ & 0.009 \\
Platelet $\left(/ \mathrm{mm}^{3}\right)$, median $($ range $)$ & $27.6(3.4-97.1)$ & $0.3(0.0-14.0)$ & $<0.0001$ \\
C-reactive protein $(\mathrm{mg} / \mathrm{dl})$, median $($ range $)$ & $0.05(0.0-11.6)$ &
\end{tabular}

P-values were calculated using Mann-Whitney U or Chi-square tests.

Table II. Stepwise multivariate logistic regression analysis of factors associated with preoperative elevation of plasma D-dimer levels in patients with gynecological cancer.

\begin{tabular}{|c|c|c|c|c|}
\hline \multirow[b]{2}{*}{ Variable } & \multirow[b]{2}{*}{ Comparison } & \multicolumn{3}{|c|}{ Multivariate } \\
\hline & & Odds ratio & P-value & $95 \% \mathrm{CI}$ \\
\hline Age & Old:Young & 1.049 & $<0.001$ & $1.029-1.069$ \\
\hline Blood type & Non-O:O & 1.602 & 0.092 & $0.926-2.774$ \\
\hline Leukocyte & High:Low & 1.000 & 0.068 & $1.000-1.000$ \\
\hline Hemoglobin & High:Low & 0.862 & 0.043 & $0.747-0.995$ \\
\hline Platelet & High:Low & 1.001 & 0.959 & $0.975-1.027$ \\
\hline C-reactive protein & High:Low & 1.250 & 0.007 & $1.063-1.471$ \\
\hline
\end{tabular}

CI, confidence interval.

Measurement of D-dimer levels and detection of VTE. Preoperative plasma D-dimer levels were measured no longer than 4 weeks prior to surgery. Informed consent was obtained from each patient prior to blood collection. The latex photometric immunoassay system was utilized and the amount of D-dimer was measured using LPIA-ACE D-D dimer II (Mitsubishi Chemical Medience Corporation, Tokyo, Japan) as the reagent. Inter-assay variability (coefficient of variation) was $<10 \%$. Primary preoperative screening for VTE was performed using meticulous evaluation of the clinical signs and plasma D-dimer levels. Clinical signs of VTE evaluated in the present study were leg swelling, tenderness along the distribution of deep veins, acute cardiovascular dysfunction, dyspnea, chest pain and loss of consciousness. Patients whose plasma D-dimer concentration exceeded pre-set cut-off values $(\geq 1.3 \mu \mathrm{g} / \mathrm{ml})$ and those who revealed clinical signs of VTE underwent computed tomography scanning of the chest, abdomen and lower extremities using multidetector row computed tomography (Toshiba, Tokyo, Japan).
Clinicopathological, hematological and biochemical data were obtained from the patients' medical records.

Statistical analysis. Chi-square and Mann-Whitney U tests and logistic regression analysis were used to investigate the correlation between various variables and plasma D-dimer levels or the occurrence of VTE. Preoperative factors associated with elevated D-dimer levels and VTE were investigated using univariate and multivariate analyses. $\mathrm{P}<0.05$ was considered to indicate a statistically significant difference.

\section{Results}

Plasma D-dimer level and associated factors. Plasma D-dimer levels were elevated $(\geq 1.3 \mu \mathrm{g} / \mathrm{ml})$ in $127 / 456$ patients (27.9\%). The incidence of elevated D-dimer levels was identified to be significantly higher in patients with ovarian cancer (57.9\%), compared with those with endometrial (24.7\%) and cervical cancer $(8.3 \% ; \mathrm{P}<0.0001)$. Results of univariate 
Table III. Analysis of risk factors associated with preoperative VTE in patients with gynecological cancer.

\begin{tabular}{|c|c|c|c|}
\hline Variables & $\operatorname{VTE}(-), n=419$ & $\operatorname{VTE}(+), \mathrm{n}=37$ & P-value \\
\hline Age (years), median (range) & $55(23-85)$ & $63(38-79)$ & $<0.0001$ \\
\hline Body mass index $\left(\mathrm{kg} / \mathrm{m}^{2}\right)$, median (range) & $22.2(13.5-46.5)$ & $22.5(14.4-27.7)$ & 0.670 \\
\hline Comorbidity, n (\%) & $111(265)$ & $18(48.6)$ & 0.004 \\
\hline Smoking, n (\%) & $36(8.6)$ & $1(2.7)$ & 0.209 \\
\hline Blood group, n (\%) & & & 0.741 \\
\hline $\mathrm{O}$ & $103(24.6)$ & $10(27.0)$ & \\
\hline Non-O & $316(75.4)$ & $27(73.0)$ & \\
\hline Leukocyte $\left(/ \mathrm{mm}^{3}\right)$, median (range) & $6300(2090-18300)$ & $6400(3840-11730)$ & 0.284 \\
\hline Hemoglobin (g/dl), median (range) & $12.9(2.9-15.7)$ & $12.6(9.0-15.2)$ & 0.066 \\
\hline Platelet $\left(/ \mathrm{mm}^{3}\right)$, median (range) & $27.8(3.4-97.1)$ & $30.8(17.5-55.5)$ & 0.069 \\
\hline C-reactive protein $(\mathrm{mg} / \mathrm{dl})$, median (range) & $0.06(0.0-14.0)$ & $0.5(0.0-14.0)$ & 0.0005 \\
\hline
\end{tabular}

P-values were calculated using Mann-Whitney U or Chi-square test. VTE, venous thromboembolism.

Table IV. Analysis of risk factors associated with preoperative VTE in patients with ovarian cancer.

\begin{tabular}{|c|c|c|c|}
\hline Variables & $\operatorname{VTE}(-), n=91$ & $\operatorname{VTE}(+), \mathrm{n}=23$ & P-value \\
\hline Age (years), median (range) & $56(30-81)$ & $61(38-79)$ & 0.099 \\
\hline Body mass index $\left(\mathrm{kg} / \mathrm{m}^{2}\right)$, median (range) & $22.0(15.0-35.6)$ & $22.4(14.4-27.7)$ & 0.919 \\
\hline Stage, $\mathrm{n}(\%)$ & & & 0.884 \\
\hline I & $36(39.5)$ & $7(30.4)$ & \\
\hline II & $7(7.7)$ & $2(8.7)$ & \\
\hline III & $38(41.8)$ & $11(47.8)$ & \\
\hline IV & $10(11.0)$ & $3(13.1)$ & \\
\hline Histology, n (\%) & & & 0.429 \\
\hline Clear & $17(18.7)$ & $6(26.1)$ & \\
\hline Non-clesr & $74(81.3)$ & $17(73.9)$ & \\
\hline Ascites, n (\%) & & & 0.0003 \\
\hline Not massive & $71(78.0)$ & $9(39.1)$ & \\
\hline Massive & $20(12.0)$ & $14(60.9)$ & \\
\hline Tumor size (cm), median (range) & $8.7(2.0-26.0)$ & $9.5(3.0-20.0)$ & 0.918 \\
\hline Comorbidity, n (\%) & $19(20.9)$ & $11(47.8)$ & 0.009 \\
\hline Smoking, n (\%) & $5(5.5)$ & $1(4.3)$ & 0.826 \\
\hline Blood group, n (\%) & & & 0.554 \\
\hline $\mathrm{O}$ & $15(16.5)$ & $5(21.7)$ & \\
\hline Non-O & $76(83.5)$ & $18(78.3)$ & \\
\hline Leukocyte $\left(/ \mathrm{mm}^{3}\right)$, median (range) & $6560(2920-17770)$ & $6400(3840-11730)$ & 0.935 \\
\hline Hemoglobin (g/dl), median (range) & $12.6(8.7-15.3)$ & $12.5(9.0-14.4)$ & 0.325 \\
\hline Platelet $\left(/ \mathrm{mm}^{3}\right)$, median (range) & $33.4(15.7-89.1)$ & $32.6(17.5-55.0)$ & 0.563 \\
\hline C-reactive protein (mg/dl), median (range) & $0.2(0.0-14.0)$ & $1.22(0.02-3.94)$ & 0.032 \\
\hline CA125, median (range) & $234.5(9.4-18238)$ & $307.6(66.8-11159)$ & 0.041 \\
\hline
\end{tabular}

P-values were calculated using Mann-Whitney U or Chi-square tests. VTE, venous thromboembolism; CA125, cancer antigen 125.

analysis indicated that age, blood type, leukocyte count, hemoglobin, platelet count and C-reactive protein (CRP) levels were associated with elevated D-dimer levels (Table I). Logistic multivariate regression revealed that advanced age, elevated CRP and low hemoglobin levels were independent factors associated with preoperative elevated plasma D-dimer levels (Table II). 
Table V. Analysis of risk factors associated with preoperative VTE in patients with endometrial cancer.

\begin{tabular}{|c|c|c|c|}
\hline Variables & $\operatorname{VTE}(-), \mathrm{n}=188$ & $\operatorname{VTE}(+), \mathrm{n}=10$ & P-value \\
\hline Age (years), median (range) & $57(23-85)$ & $69.5(44-76)$ & 0.044 \\
\hline Body mass index $\left(\mathrm{kg} / \mathrm{m}^{2}\right)$, median (range) & $22.5(13.5-46.5)$ & $22.5(20.8-26.3)$ & 0.444 \\
\hline Stage, n $(\%)$ & & & 0.040 \\
\hline $\mathrm{I}+\mathrm{II}$ & $147(78.2)$ & $5(50.0)$ & \\
\hline III+IV & $41(21.8)$ & $5(50.0)$ & \\
\hline Histology, n (\%) & & & 0.752 \\
\hline Type 1 & $122(64.9)$ & $6(60.0)$ & \\
\hline Type 2 & $66(35.1)$ & $4(40.0)$ & \\
\hline Comorbidity, n (\%) & $116(61.7)$ & $5(50.0)$ & 0.460 \\
\hline Smoking, n (\%) & $15(8.0)$ & $0(0.0)$ & 0.353 \\
\hline Blood group, n (\%) & & & 0.376 \\
\hline $\mathrm{O}$ & $51(27.1)$ & $4(40.0)$ & \\
\hline Non-O & $137(62.9)$ & $6(60.0)$ & \\
\hline Leukocyte $\left(/ \mathrm{mm}^{3}\right)$, median (range) & $6260(2090-18300)$ & $6670(5110-10830)$ & 0.537 \\
\hline Hemoglobin (g/dl), median (range) & $12.9(2.9-15.9)$ & $13.1(9.3-15.2)$ & 0.808 \\
\hline Platelet $\left(/ \mathrm{mm}^{3}\right)$, median (range) & $26.7(3.4-97.1)$ & $27.9(22.0-46.6)$ & 0.247 \\
\hline C-reactive protein $(\mathrm{mg} / \mathrm{dl})$, median (range) & $0.06(0.0-9.5)$ & $0.07(0.02-2.54)$ & 0.333 \\
\hline
\end{tabular}

P-values were calculated using Mann-Whitney U or Chi-square tests. VTE, venous thromboembolism.

Table VI. Analysis of risk factors associated with preoperative VTE in patients with cervical cancer.

\begin{tabular}{|c|c|c|c|}
\hline Variables & $\operatorname{VTE}(-), n=140$ & $\operatorname{VTE}(+), n=4$ & P-value \\
\hline Age (years), median (range) & $43.5(23-85)$ & $58.5(44-76)$ & 0.039 \\
\hline Body mass index $\left(\mathrm{kg} / \mathrm{m}^{2}\right)$, median (range) & $21.6(15.6-29.9)$ & $22.9(15.6-26.1)$ & 0.799 \\
\hline Stage, $\mathrm{n}(\%)$ & & & 0.920 \\
\hline I & $108(77.1)$ & $3(75.0)$ & \\
\hline II & $32(22.9)$ & $1(25.0)$ & \\
\hline Histology, n (\%) & & & 0.041 \\
\hline $\mathrm{SCC}$ & $101(72.1)$ & $1(25.0)$ & \\
\hline Non-SCC & $39(27.9)$ & $3(75.0)$ & \\
\hline Comorbidity, n (\%) & $20(14.3)$ & $2(50.0)$ & 0.050 \\
\hline Smoking, n (\%) & $18(12.9)$ & $0(0.0)$ & 0.464 \\
\hline Blood group, n (\%) & & & 0.949 \\
\hline $\mathrm{O}$ & $37(26.4)$ & $1(25.0)$ & \\
\hline Non-O & $103(73.6)$ & $3(75.0)$ & \\
\hline Leukocyte $\left(/ \mathrm{mm}^{3}\right)$, median (range) & $6110(3180-15140)$ & $7040(4700-9990)$ & 0.572 \\
\hline Hemoglobin (g/dl), median (range) & $13.0(5.6-15.1)$ & $12.8(12.6-13.2)$ & 0.702 \\
\hline Platelet $\left(/ \mathrm{mm}^{3}\right)$, median (range) & $27.7(9.8-66.9)$ & $29.6(25.3-33.4)$ & 0.527 \\
\hline C-reactive protein (mg/dl), median (range) & $0.04(0.0-11.6)$ & $0.07(0.0-0.09)$ & 0.191 \\
\hline
\end{tabular}

P-values were calculated using Mann-Whitney U or Chi-square tests. VTE, venous thromboembolism.

Preoperative VTE. In the present study population, VTE was detected in $37(8.1 \%)$ patients prior to surgery. The incidence of VTE in patients with ovarian, endometrial and cervical cancer was $20.2,5.1$ and $2.8 \%$, respectively. The incidence of preoperative VTE in patients with ovarian cancer was revealed to be significantly higher than in patients with endometrial or cervical cancer $(\mathrm{P}<0.0001)$. PTE was identified in 18 patients $(3.9 \%)$. The incidence of PTE in patients with ovarian, endometrial and cervical cancer was 8.8, 3.0 and $1.4 \%$, respectively. The incidence of preoperative PTE in 
patients with ovarian cancer was identified to be significantly higher than in patients with endometrial or cervical cancer $(\mathrm{P}=0.028$ and 0.006 , respectively). One patient with PTE was clinically symptomatic, with dyspnea, but PTE was not fatal in this case. A substantial number of thrombi and PTEs were treated with anticoagulation therapy using parenteral unfractionated heparin prior to surgery. Insertion of an inferior vena cava filter was required in 5 patients.

Risk factors for preoperative VTE in gynecological cancer. Results of univariate analysis indicated that age, comorbidity and CRP were significant risk factors for preoperative VTE (Table III). Logistic regression multivariate analysis revealed that advanced age was an independent risk factor for the occurrence of preoperative VTE [hazard ratio (HR), 1.049; 95\% CI, 1.016-1.083; $\mathrm{P}=0.003$ ].

Risk factors for preoperative VTE in ovarian, endometrial and cervical cancers. Massive ascites, the presence of comorbidities and increased CRP and cancer antigen 125 levels were identified to be significant risk factors in patients with ovarian cancer (Table IV). Logistic regression multivariate analysis revealed that the presence of massive ascites (HR, 7.017; 95\% CI, 2.293-22.025; $\mathrm{P}=0.0007)$ and comorbidities (HR, 3.696; 95\% CI, 1.289-10.598; $\mathrm{P}=0.015$ ) were independent risk factors for the occurrence of preoperative VTE. Age and stage of malignancy were significant risk factors in patients with endometrial cancer (Table V). Logistic regression multivariate analysis revealed that advanced age (HR, 1.072; 95\% CI, 1.003-1.146; $\mathrm{P}=0.041)$ and stage (HR, 4.394; 95\% CI, 1.144-16.879; $\mathrm{P}=0.031$ ) were independent risk factors for the occurrence of preoperative VTE. Advanced age and the identification of non-squamous cell cancer by histological analysis were significant risk factors in patients with cervical cancer (Table VI). Logistic regression multivariate analysis revealed that advanced age was an independent risk factor for the occurrence of preoperative VTE (HR, 1.169; 95\% CI, 1.009-1.354; $\mathrm{P}=0.038$ ).

\section{Discussion}

Cancer is a significant risk factor for VTE. Surgery, a first-line treatment of gynecological cancers, is an additional risk factor for VTE. Therefore, preoperative screening for VTE is important. In the present study, D-dimer levels were used to screen for preoperative VTE, including asymptomatic/subclinical VTE, in Japanese females with invasive gynecological cancer. Elevated D-dimer levels are not a specific indicator of VTE. Increased levels of D-dimer have also been observed in individuals of advanced age, during pregnancy, following surgery and trauma, inflammatory states, infections and specific types of cancer (4). In addition, false-positive increases in D-dimer levels are common in cancer patients. Therefore, the specificity and positive predictive value of the assay is likely to be reduced in cancer patients. There are a number of assays for D-dimer levels and cut-off values depend on the assay. The negative predictive value for VTE has been reported as 100\% using a cut-off value of $1.2 \mu \mathrm{g} / \mathrm{ml}$ and a positive predictive value $>50 \%$ using a cut-off value of $3 \mu \mathrm{g} / \mathrm{ml}$ has been reported with the assay used in the current study $(5,6)$. In general, a positive predictive value $>50 \%$ is effective for diagnosis. Therefore, in the current study, a diagnosis of preoperative VTE was considered in patients whose plasma $\mathrm{D}$-dimer concentration $>1.3 \mu \mathrm{g} / \mathrm{ml}$.

Patients with high D-dimer levels are likely to be in a hypercoagulable state. It was previously reported that increased D-dimer concentrations positively correlate with the occurrence of VTE risk (7). The current study demonstrated that the incidence of elevated D-dimer levels was significantly higher in patients with ovarian cancer, compared with those with endometrial and cervical cancers. Furthermore, results revealed that old-age, non-O blood type, low hemoglobin level, elevated platelet and leukocyte counts and elevated CRP levels were significant factors associated with elevated D-dimer levels.

Increased plasma levels of factors VIII (FVIII) and IX (FIX) have been reported to be independent risk factors for VTE (8). It is well known that FIX levels increase with age (9). In addition, individuals with non- $\mathrm{O}$ blood groups are at a significantly increased risk of arterial thromboembolic disease and VTE $(10,11)$. According to the ABO blood group system, blood group is determined by plasma levels of 2 coagulation glycoproteins, von Willebrand factor (VWF) and FVIII. VWF and FVIII are found in circulation in normal plasma as a noncovalent complex and are important for normal hemostasis. Compared with non-O group individuals, those with blood group $\mathrm{O}$ were revealed to have significantly reduced plasma VWF and FVIII levels. This explains why individuals with clinical thrombosis have reduced levels of these factors (12). We previously reported that the non-O blood group was an independent risk factor for postoperative VTE in patients with gynecological cancer (13). These results, in combination with the current observations, indicate that elderly cancer patients with a non-O blood group must be considered to have a hypercoagulable state.

CRP is a downstream marker of inflammation and is considered a predictor of cardiovascular events and mortality (14). A prospective study on 507 cancer patients reported that elevated CRP levels correlated with the development of VTE (15). Moreover, additional studies have demonstrated that elevated platelet and leukocyte counts and low hemoglobin levels were associated with VTE in cancer patients (14). There is evidence that pre-chemotherapy thrombocytosis, leukocytosis and low hemoglobin levels are predictive biomarkers of VTE in patients receiving chemotherapy. Notably, low hemoglobin and elevated platelet and leukocyte counts were associated with a preoperative increase in D-dimer levels in the present study.

This study further investigated the incidence and risk factors associated with preoperative VTE. Results demonstrate that $20 \%$ patients with ovarian cancer have VTE prior to surgery, consistent with results of a previous study (16). Incidence of preoperative VTE was revealed to be significantly higher in patients with ovarian cancer compared with those with other gynecological cancers. Therefore, preoperative screening of patients with ovarian cancer is particularly important.

Advanced age was identified to be an independent risk factor for the occurrence of preoperative VTE in gynecological cancer. Advanced age, a common characteristic of a number of cancer patients, correlated with an increased risk of VTE. In ovarian cancer, massive ascites and the presence 
of comorbidities were also independent risk factors for preoperative VTE. It was previously reported that the presence and number of comorbidities affect the risk of VTE. In patients with ovarian cancer, HRs for the development of VTE increase from 2.1 in patients with a single comorbidity to 3.9 in patients with 3 comorbidities (17). Satoh et al reported that massive ascites and clear cell histology were significantly associated with preoperative DVT, although clear cell histology was not associated with VTE in the present study (16). In endometrial cancer, advanced age and stage of malignancy were independent risk factors for the occurrence of preoperative VTE. The same research group reported that advanced stage and non-endometrioid histology were significantly associated with preoperative VTE, although the histological observations were not associated with VTE in the present study (18). In cervical cancer, advanced age and non-squamous cell carcinoma were risk factors for preoperative VTE. Although histology was not an independent factor in the current study, squamous cell carcinoma, the most common histological form of cervical cancer, appears to be associated with a lower risk of VTE than adenocarcinoma (19).

Results from the current study demonstrate that high plasma D-dimer levels and the incidence of VTE prior to surgery were higher in patients with ovarian cancer compared with those with other gynecological cancers. Furthermore, this study demonstrated, through multivariate analysis, that advanced age and low hemoglobin and elevated CRP levels were significant risk factors for a preoperative increase in plasma D-dimer levels and that advanced age was an independent risk factor for preoperative VTE in gynecological cancer.

\section{References}

1. Lin A, Ryu J, Harvey D, et al: Low-dose warfarin does not decrease the rate of thrombosis in patients with cervix and vulvo-vaginal cancer treated with chemotherapy, radiation and erythropoietin. Gynecol Oncol 102: 98-102, 2006.

2. Einstein MH, Pritts EA and Hartenbach EM: Venous thromboembolism prevention in gynecologic cancer surgery: a sysytematic review. Gynecol Oncol 105: 813-819, 2007.
3. NCCN Clinical Practice Guidelines in Oncology: Venous thromboembolic disease, Version 2. National Comprehensive Cancer Network, Fort Washington, PA, 2011.

4. Hunt BJ: Plasma D-dimer assays in the diagnosis of venous thromboembolism in cancer patients. J Thromb Haemost 4: 50-51, 2006.

5. Nomura $\mathrm{H}$, Wada $\mathrm{H}$, Mizuno T, et al: Negative predictive value of D-dimer for diagnosis of venous thromboembolism. Int $\mathrm{J}$ Hematol 87: 250-255, 2008

6. Wada H, Kobayashi T, Abe Y, et al: Elevated levels of soluble fibrin or D-dimer indicate high risk of thrombosis. J Thromb Haemost 4: 1253-1258, 2006.

7. Cushman M, Folsom AR, Wang L, et al: Fibrin fragment D-dimer and the risk of future venous thrombosis. Blood 101: 1243-1248, 2003.

8. Bertina RM: Elevated clotting factor levels and venous thrombosis. Pathophysiol Haemost Thromb 33: 395-400, 2003/2004.

9. Sweenery JD and Hoerning LA: Age-dependent effect on the level of factor IX. Am J Clin Pathol 99: 687-688, 1993.

10. O'Donnell J and Laffan MA: The relationship between ABO hist-blood group, factor VIII and von Willebrand factor. Transfus Med 11: 343-351, 2001.

11. Koster T, Blann AD, Brief E, et al: Role of clotting factor VIII in effect of von Willebrand factor on occurrence of deep-vein thrombosis. Lancet 345: 152-155, 1995.

12. Jenkins PV and O'Donnell JS: ABO blood group determines plasma von Willebrand factor levels: a biologic function after all? Transfusion 46: 1836-1844, 2006.

13. Kodama J, Seki N, Masahiro S, et al: D-dimer level as a risk factor for postoperative venous thromboembolism in Japanese women with gynecologic cancer. Ann Oncol 21: 1651-1656, 2010.

14. Khorana AA and Connolly GC: Assessing risk of venous thromboembolism in the cancer patients. J Clin Oncol 27: 4839-4847, 2009.

15. Kröger K, Weiland D, Ose C, et al: Risk factors for venous thromboembolic events in cancer patients. Ann Oncol 17: 297-303, 2006.

16. Satoh T, Oki A, Uno K, et al: High incidence of silent venous thromboembolism before treatment in ovarian cancer. Brit J Cancer 97: 1053-1057, 2007.

17. Rodriguez AO, Wun T, Chew $\mathrm{H}$, et al: Venous thromboembolism in ovarian cancer. Gynecol Oncol 105: 784-790, 2007.

18. Satoh T, Mastumoto K, Uno K, et al: Silent venous thromboembolism before treatment in endometrial cancer and the risk factors. Brit J Cancer 99: 1034-1039, 2008.

19. Barbera L and Thomas G: Venous thromboembolism in cervical cancer. Lancer Oncol 9: 54-60, 2008. 